\title{
20 - Fatores que determinam a ocorrência de formigas, em particular poneromorfas, no dossel de florestas tropicais
}

\author{
Wesley Duarte DaRocha \\ Jacques H. C. Delabie \\ Frederico Siqueira Neves \\ Sérvio Pontes Ribeiro
}

\section{SciELO Books / SciELO Livros / SciELO Libros}

DAROCHA, WD., et al. Fatores que determinam a ocorrência de formigas, em particular poneromorfas, no dossel de florestas tropicais. In: DELABIE, JHC., et al., orgs. As formigas poneromorfas do Brasil [online]. Ilhéus, BA: Editus, 2015, pp. 295-312. ISBN 978-85-7455-441-9.

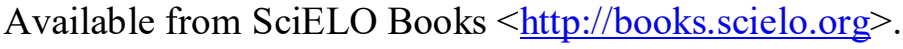

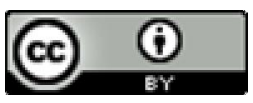

All the contents of this work, except where otherwise noted, is licensed under a Creative Commons Attribution 4.0 International license.

Todo o conteúdo deste trabalho, exceto quando houver ressalva, é publicado sob a licença $\underline{\text { Creative Commons }}$ Atribição 4.0.

Todo el contenido de esta obra, excepto donde se indique lo contrario, está bajo licencia de la licencia $\underline{\text { Creative }}$ Commons Reconocimento 4.0. 


\title{
Fatores que determinam a ocorrência de formigas, em particular poneromorfas, no dossel de florestas tropicais
}

\author{
Wesley Duarte DaRocha, Jacques H. C. Delabie, \\ Frederico Siqueira Neves, Sérvio Pontes Ribeiro
}

Resumo

O dossel das florestas tropicais abriga grande parte da diversidade animal. Nesse, as formigas se destacam devido a sua biomassa e diversidade que são notavelmente elevadas na Região Neotropical. A biomassa desses organismos no dossel das florestas é superior a de vertebrados e pode representar entre 20 e $40 \%$ dos artrópodes. A mesma diversidade e grupos funcionais são encontrados nos sistemas agroflorestais. Além de interagir com numerosos organismos, plantas ou animais, do próprio dossel, as formigas da copa das árvores podem estar sob a influência das espécies que vivem no solo, principalmente em agro- ou ecossistemas com árvores baixas. Estudos comparativos sobre os ambientes da serapilheira e do dossel vêm buscando entender os padrões para os mecanismos que estruturam as assembleias de formigas arborícolas comparativamente às daquelas que habitam o solo. Em particular, as poneromorfas arborícolas foram até então negligenciadas em estudos de comunidades de formigas arbóreas, apesar de ser um grupo quase tão diverso nesse ambiente quanto na serapilheira. As espécies arborícolas desse grupo são, em sua maioria, de grande tamanho corpóreo e apresentam grande resistência à dessecação comparadas às espécies menores que vivem no mesmo habitat, que é caracterizado por elevadas temperaturas, elevada amplitude térmica e baixa umidade relativa. Seu comportamento, principalmente predador, e com colônias de relativamente poucos indivíduos, inclui forrageio solitário durante o qual uma área extensa pode ser visitada. Esses aspectos comportamentais, suas exigências nutricionais e sua morfologia fazem com que essas formigas sejam exigentes quanto aos lugares de nidificação e forrageio, com uma frequência elevada em dosséis que mantêm epífitas e cupinzeiros abandonados que, por sua vez, são extremamente favoráveis à instalação de suas colônias. Várias hipóteses para se estudar para sobre o gradiente de condições e de recursos entre dossel e serapilheira têm sido examinadas para explicar esses padrões. Este capítulo faz uma breve abordagem sobre os aspectos ecológicos estruturais abióticos e bióticos dos ambientes de dossel comparados à serapilheira, mostrando como os aspectos microclimáticos, estruturadores de habitat e as condições nutricionais são determinantes para a sobrevida de poneromorfas no dossel. As hipóteses apresentada aqui visam entender a estruturação da comunidade de formigas que vivem em ambientes de dossel e serapilheira, com consequências sobre a morfologia dos próprios insetos.

DAROCHA, Wesley Duarte; DELABIE, Jacques H. C.; NEVES, Frederico Siqueira; RIBEIRO, Sérvio Pontes. Fatores que determinam a ocorrência de formigas, em particular poneromorfas, no dossel de florestas tropicais. In: DELABIE, Jacques H. C. et al. As formigas poneromorfas do Brasil. Ilhéus: Editus, 2015. p. 295-312. 
Factors determining the occurrence of ants, in particular poneromorphs, in the tropical forest canopy - The canopy of tropical forests houses much of animal diversity. Here, the ants stand out due to their high biomass and diversity, particularly so in the Neotropical Region. The biomass of these organisms in the forest canopy is higher than that of vertebrates and may represent between $20-40 \%$ of that of arthropods. Similar diversities and functional groups can be found in many Neotropical agroforestry systems. Besides interacting with numerous organisms, plants or animals, and the canopy itself, the ants from the treetops may be under the influence of species living in or on the ground, especially in agroecosystems or ecosystems with low trees. Comparative studies on litter and canopy environments are seeking to understand the mechanisms that structure the arboreal ant assemblages compared to those inhabiting the ground. In particular, arboreal poneromorphs have been neglected in most of the studies on tree ant assemblages, despite this being is a group that is almost as diverse in this environment as in the litter. The arboreal species from this group are mostly of large body size and have good resistance to desiccation compared to smaller species living in the same habitat, which is characterized by high temperatures, high thermal amplitudes and low relative humidity. Their behaviour is mostly predatory, and their colonies are of relatively few individuals, generally exhibiting solitary foraging during which a large area can be visited. These behavioral aspects, their nutritional requirements and their morphology tend to dictate the favourability of their nesting and foraging places. A high frequency of poneromorph nests is found in canopies with epiphytes and abandoned termite nests, as these are extremely favourable for the installation of their colonies. Several hypotheses aiming to study the gradient of conditions and resources between canopy and litter have been examined to explain these patterns. This chapter gives a brief review of the abiotic, structural and ecological aspects of the canopy habitats in comparison to that of the litter, indicating how microclimate, habitat structuring and nutritional conditions could be decisive for poneromorph survival in the canopy. The hypotheses presented here aim to help us to understand the structure of the ant community living in canopy and litter environments, and the consequences for the morphology of the insects themselves.

\section{Introdução}

A copa das árvores (o dossel) das florestas tropicais pode abrigar grande parte da diversidade de animais de uma floresta (ERWIN, 1982; NOVOTNY et al., 2002) dentre os animais presentes no dossel, as formigas se destacam devido a sua elevada diversidade e biomassa. A diversidade elevada das formigas arbóreas é notória na Região Neotropical (WILSON, 1987; TOBIN, 1995; YANOVIAK et al., 2011). A biomassa desses organismos no dossel de florestas tropicais é superior a de vertebrados (WILSON, 1987) e pode representar entre 20 e $40 \%$ da biomassa total dos artrópodes (TOBIN, 1995).

O termo dossel corresponde por definição à região superior de uma floresta, constituído pelo conjunto das copas de árvores, diferindo em termos de espécies, tamanho, arquitetura, fenologia (perenes, floração, etc.) e idade (HALLÉ; OLDEMAN; TOMLINSON, 1978; LAWTON, 1983; BELL; BELL; DINES, 1999), além de toda a estrutura associada a estas, como madeiras vivas e mortas, folhas, epífitas, lianas, solos suspensos e microclimas (NADKARNI, 1995; PAOLETTI et al., 1991; BASSET et al., 2003; YANOVIAK et al., 2011; YANOVIAK, 2015).

No dossel das florestas tropicais uma grande diversidade de espécies que compõem diferentes grupos funcionais (ver ANDERSEN, 1995; NEVES et al., 2013; BRANDÃO et al., 2012) da comunidade de formigas divide o espaço formado pelo dossel: o primeiro é constituído pelas formigas arborícolas sensu stricto, que dependem sobretudo da árvore hospedeira e dos recursos encontrados nela; o segundo corresponde às espécies que vivem 
associadas às epífitas que retêm nas suas raízes matéria orgânica em decomposição, estruturando um substrato que constitui um verdadeiro solo, o "solo suspenso" (ver PAOLETTI et al., 1991; DELABIE, 2003; DaROCHA et al., 2015); o terceiro é formado pela fauna que vive nos "jardins de formigas" (YU, 1994; DELABIE; OSPINA; ZABALA, 2003; DEJE$\mathrm{AN}$ et al., 2007), considerado, às vezes, uma simples variante do caso anterior. De fato, cada um desses grupos funcionais é o produto da adaptação de um segmento distinto da mirmecofauna que influencia a comunidade local de formigas. Nesses segmentos, as formigas participam de vários níveis da cadeia trófica, como predadoras, detritívoras, herbívoras de forma direta, como as formigas cortadeiras, ou indireta através da utilização de exsudatos açucarados liberados por insetos herbívoros trofobiontes ou recursos presentes nas árvores como os nectários extra-florais (NEFs) (HUNT, 2003).

As assembleias de invertebrados e vertebrados que vivem sobre as árvores podem ser afetadas pelas características estruturais da planta hospedeira sob diferentes formas. Alguns estudos demonstram que o desenvolvimento da planta hospedeira afeta diretamente a distribuição dos insetos (VASCONCELOS; DAVIDSON, 2000; LAWRENCE; POTTS; WHITHAM, 2003; FONSECA; BENSON, 2003; DJIÉTO-LORDON, 2004; CAMPOS et al., 2006; CONCEIÇÃO et al., 2014). Assim, as mudanças que ocorrem nas copas das árvores quando essas atingem os estratos superiores da vegetação podem causar modificações nas condições do habitat afetando a fauna de insetos associados (BASSET; ABERLENC; DELVARE, 1992; CAMPOS et al., 2006; NEVES et al., 2013, 2014). No entanto, o efeito do aumento da disponibilidade de alimentos e abrigos específicos sobre a mirmecofauna em relação ao crescimento da planta ainda é pouco estudado, e o conhecimento correspondente é restrito a mirmecófitas (VASCONCELOS; DAVIDSON, 2000; CAMPOS et al., 2006; COSTA et al., 2012).

As associações formiga-planta já motivaram alguns dos estudos sobre possíveis mutualismos (BENSON, 1985; DAVIDSON; MACKEY, 1993; JOVILET, 1996). Embora o grau de dependência recíproca varie de uma associação à outra, em situações extremas, as formigas podem retirar toda sua alimentação da planta hospedeira na forma de néctar, rico em carboidrato, além de a mesma lhes oferecer locais de nidificação. As formigas, em tro$\mathrm{ca}$, defendem as plantas contra herbívoros e fornecem nutrientes na forma de rejeitos orgânicos e fezes, ricos em ureia, além de as auxiliarem na dispersão de suas sementes. Em raríssimos casos, as formigas podem também atuar como polinizadoras (BEATTIE, 1985; FONSECA, 1994; TOBIN, 1995).

Estas associações entre formigas e plantas se tornam mais complexas em dosséis florestais do que em agrossistemas. Componentes sutis do habitat e que derivam da ontogênese da planta hospedeira aparentemente determinam a riqueza e abundância das espécies de formigas, conjuntamente com a presença de estruturas mirmecófilas e recursos alimentares (CAMPOS et al., 2006; DELABIE et al., 2010; FEITOSA et al., 2012; REIS et al., 2013; CONCEIÇÃO et al., 2014). Em contrapartida, as formigas, bem como outros invertebrados, transportam nutrientes e detritos para bifurcações de troncos, fustes e outros locais da copa. No entanto, o solo suspenso é formado, sobretudo, pela acumulação natural de detritos orgânicos oferecendo recursos para diversos organismos (ver PAOLETTI et al., 1988). Este transporte é responsável pelo estabelecimento de diversas espécies de epífitas ou por um mecanismo de enriquecimento de microhabitat quando já ocorrem epífitas associadas (PAOLETTI et al., 1988; DaROCHA et al., 2015).

Essa grande diversidade de espécies e os mesmos grupos funcionais são também encontrados nos sistemas agroflorestais (LESTON, 1978; MAJER; DELABIE; SMITH, 1994; DaROCHA et al., 2015). Esses sistemas possuem uma dinâmica baseada na prática de manejo dos recursos naturais, o que diversifica a produção e aumenta os benefícios sociais, econômicos e ambientais através da integração do uso e do manejo das árvores e outras plantas lenhosas nas atividades típicas de produção da fazenda e nas paisagens agrícolas (ICRAF 2000). Na Região Neotropical, encontramos como exemplos de sistemas agroflorestais os cacauais do sudeste da Bahia. Grandes extensões de terra abrigam predominantemente esses sistemas que mantêm parte das árvores remanescentes da mata nativa para sombreamento da lavoura cacaueira, conhecidos regionalmente como cabruca (DELABIE et al., 2007; CASSANO et al., 2009). As cabrucas têm um importante papel na conservação da biodiversidade regional e constituem ótimos habitats para estudar esses componentes que interagem de forma complexa e que participam na estruturação do mosaico de espécies de formigas presentes no dossel (LESTON, 1978; MAJER; DELABIE; SMITH, 1994; RIBEIRO et al., 2013). Com 
alta diversidade nesses agrossistemas (PERFECTO et al., 1996; DELABIE et al., 2007), as formigas arborícolas são frequentemente predadores generalistas ou especializados, ou ainda simples espécies oportunistas que podem atuar como agentes de controle biológico na regulação das populações de fitófagos que se alimentam das plantas cultivadas, pela predação direta ou outros mecanismos (WAY; KHOO, 1992; ROBERTS; COOPER; PETIT, 2000).

Além das interações que ocorrem no dossel, a fauna de formigas associada à copa das árvores pode estar sob influência das formigas presentes no solo, principalmente em sistemas que apresentam árvores baixas (MARTINEZ, 2015). Estudos comparativos nos diferentes ambientes da serapilheira e do dossel vêm buscando entender os padrões que estruturam as assembleias de formigas arborícolas comparativamente às das que vivem no solo (BASSET et al., 2012). Várias hipóteses para estudar o gradiente de condições e recursos entre dossel e serapilheira têm sido examinadas para explicar esses padrões. A Tabela 20.I apresenta hipóteses consistentes para a estruturação da comunidade de formigas que vivem em ambientes de dossel e serapilheira, e isso inclui também consequências sobre a morfologia dos próprios insetos.

Dentre as formigas presentes no dossel de florestas tropicais ou agroecossistemas, ainda se conhece pouco a respeito da distribuição das espécies do grupo das poneromorfas e dos mecanismos determinantes dessa distribuição. A escassez de informações sobre essas formigas de comportamento principalmente predador (JIMÉNEZ et al., 2008) se deve sobretudo à dificuldade de acesso dos pesquisadores ao estrato dossel. Porém, estudos específicos para alguns gêneros arborícolas e das particularidades das formigas que utilizam os microhabitats bromelícolas presentes no dossel (DaROCHA et al., 2015) têm contribuído ao conhecimento da ecologia das poneromorfas arborícolas.

Assim, entender o papel potencial de alguns mecanismos, microclima, estrutura do habitat e disponibilidade de nutrientes sobre a distribuição da comunidade de formigas de dossel é essencial para predizer futuros padrões da diversidade nos dosséis tropicais, assim como de determinados grupos de formigas ainda pouco estudados, como, por exemplo, as poneromorfas.

\section{Influência do microclima nas comunidades de formigas do dossel}

O dossel das florestas tropicais apresenta condições microclimáticas drasticamente diferentes dos estratos inferiores, como a serapilheira. $\mathrm{Na}$ serapilheira existe uma relação entre microclima e biodiversidade, enquanto que, no dossel, essa relação difere em função dos táxons considerados. $\mathrm{O}$ microclima está correlacionado com a diversidade de formigas, e temperatura e precipitação representam frequentemente os dois mecanismos que melhor determinam a diversidade de formigas que vivem na serapilheira (KASPARI; WARD; YUAN, 2004; DUNN et al., 2009). Entretanto, o dossel florestal apresenta temperaturas mais elevadas e umidade relativa baixa, e segundo Hood e Tschinkel (1990), as formigas arborícolas são mais expostas às variações climáticas do que as formigas que forrageiam na serapilheira. Portanto, a comunidade de formigas associada às copas das arbóreas pode estar mais fortemente associada às variações de precipitação e temperatura do que a de formigas presentes na serapilheira (KASPARI et al., 2014).

Aspectos relacionados à desidratação foram levantados nos estudos de HOOD e TSCHINKEL (1990), que comparam a perda de água e resistência à dessecação entre as formigas arborícolas e as do deserto, sugerindo que o habitat arbóreo é ao menos tão estressante como o do deserto. Diante de panorama da dessecação, espécies maiores parecem apresentar uma maior resistência à desidratação e uma ampla tolerância térmica (WEISER et al., 2010; KASPARI et al., 2014). No ambiente de dossel é comum observar formigas de grande e médio portes forrageando e caminhando rapidamente sobre troncos e galhos em busca de alimento, mesmo

TABELA 20.I - Hipóteses testadas, não mutualmente exclusivas, para explicar os padrões observados nas assembleias de formigas em estudos comparativos entre os ambientes de dossel e serapilheira

\begin{tabular}{l|l}
\hline \multicolumn{1}{c}{ Hipóteses } & \multicolumn{1}{c}{ Referências } \\
\hline 1. Condições do clima & (WEISER et al., 2010; KASPARI et al., 2014) \\
\hline 2. Estrutura do habitat & (YANOVIAK; KASPARI, 2000; YANOVIAK et al., 2012) \\
\hline 3. Limitação por nutrientes & (KASPARI; YANOVIAK, 2001, 2009) \\
\hline
\end{tabular}


em períodos quentes, enquanto que, nos estratos inferiores, as espécies são em geral menores (KASPARI et al., 2014). Em diferentes estudos, a correlação entre morfologia das formigas (tamanho corporal) e as condições climáticas (por exemplo, temperatura) apontam para uma relação positiva, sendo que as formigas do dossel são em média quatro vezes maiores que as espécies presentes nos estratos inferiores (KASPARI et al., 2014).

Dentro da comunidade de formigas arbóreas, o complexo poneromorfas inclui espécies de maior tamanho corporal como, por exemplo, Gnamptogenys concinna (Fr. Smith, 1858) (Figura 20.1a), Anochetus hohenbergiae Feitosa; Delabie, 2012 (Figura 20.1b) e Neoponera villosa (Fabricius, 1804) (Figura 20.1c), que, segundo a literatura, são as mais bem adaptadas a viver nesse ambiente com elevada amplitude térmica. Por sua vez, o gradiente de umidade não tem efeito aparente sobre o tamanho corporal das formigas (ver KASPARI, 1993) e as formigas de maior tamanho corporal forrageiam indiferentemente da variação de umidade: elas são generalistas microclimáticas. Por sua vez, as espécies menores utilizam estruturas como túneis ou ocos de galhos como trilhas para manter a atividade de forrageio em microclimas secos.

\section{Importância da estrutura do habitat nas comunidades de formigas arbóreas}

Aspectos estruturais de um ambiente podem ser tratados como mecanismos determinantes de uma comunidade. Em particular, cada componente estrutural de um habitat, por exemplo, galhos, lianas e epífitas, são fundamentais para a estruturação da comunidade de formigas arbóreas (YANOVIAK et al., 2011, 2012; YANOVIAK, 2015), assim como os troncos caídos (DELABIE et al., 1997), folhas e espessura da serapilheira (KASPARI; WEISER, 1999) são para as formigas da serapilheira. Assim, os mecanismos estruturadores das assembleias de formigas do dossel são diferentes dos da serapilheira. A serapilheira em uma floresta tropical úmida é um ambiente onde os recursos são mais variáveis e bastante heterogêneos em micro escala. O substrato é extremamente acidentado em pequena escala ("rugoso"), formado pelo acúmulo de folhas, detritos vegetais, com numerosos obstáculos sendo favorável, sobretudo, às formigas pequenas que forrageiam e nidificam nesse mesmo habitat. Também é favorável àquelas espécies hipogeicas que nidificam
FIGURA 20.1 - Poneromorfas arborícolas: (a) - Gnamptogenys concinna transportando uma presa (Curculionidae) a 34 metros de altura na copa de uma gindiba (Sloanea obtusifolia, Elaeocarpaceae), Parque Estadual Serra do Conduru, Uruçuca, Bahia, março de 2009; (b) - operária de Anochetus hohenbergiae observada na base de uma bromélia epífita (gênero Hohenbergia, Bromeliaceae), dossel de uma árvore de sombreamento do agrossistema cacaueiro ("cabrucas"), Una-Bahia, outubro de 2014; e (c) - Neoponera villosa, fêmea forrageando na copa de um cacaueiro (Theobroma cacao, Sterculiaceae), Una-Bahia, fevereiro de 2015 (Fotos: Wesley DaRocha).

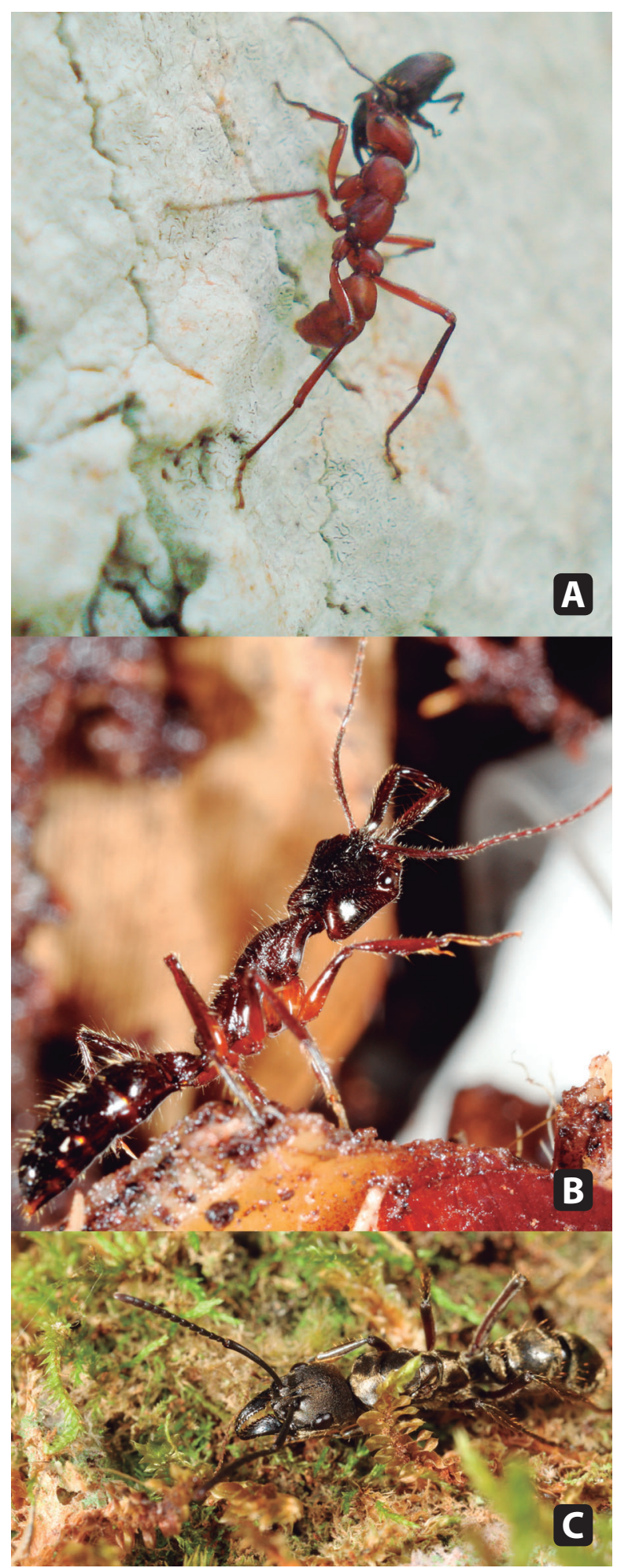


e buscam recursos no subsolo, por exemplo, ou aquelas que forrageiam em galerias abertas por outros organismos e que muitas vezes são cegas ou com poucos recursos visuais (HÖLLDOBLER; WILSON, 1990).

Já o dossel de uma floresta tropical úmida é um ambiente que apresenta recursos menos variáveis. Sua constância resulta em previsibilidade espacial; no entanto, os recursos podem ser sazonais como, por exemplo, nas florestas tropicais estacionais. Assim, são animais de grande porte que vivem no dossel e que usam a visão para forragear pelos troncos e galhos (HÖLLDOBLER, 1980). O substrato arbóreo formado pelos troncos, galhos, ramos e até mesmo epífitas pode ser considerado "linear" comparado ao substrato serapilheira, bem mais "tridimensional" comparativamente, o que permite a formação de trilhas lineares ou até a eliminação de trilhas quando as espécies fazem uso da visão (ver HÖLLDOBLER, 1980) (Figura 20.2). Várias operárias de espécies de formigas neotropicais arbóreas apresentam adaptações morfológicas que lhes permite o deslocamento vertical através de planagem na direção do tronco da árvore hospedeira após uma queda ou quando fugindo de algum predador (YANOVIAK; FISHER; ALONSO, 2008; YANOVIAK; DUDLEY; KASPARI, 2005).

Características morfológicas e estruturais da vegetação, tais como diâmetro do galho, rugosidade da casca e tamanho da árvore, são de grande importância para determinar a distribuição local e o uso dos recursos por diversas espécies de formigas arbóreas. Isso se dá em função de seus atributos morfológicos, tais como tamanho corporal, ou ainda em função das suas necessidades de nidificação, tais como ninhos instalados em galhos ocos ou construídos com matéria orgânica e encostados a um galho (DELABIE et al., 1991). Segundo Yanoviak et al. (2012), formigas de tamanhos distintos visitam tanto galhos rugosos ou lisos, visto que formigas pequenas podem se deslocar pelos sulcos dos galhos rugosos enquanto que as formigas maiores caminham sobre eles. Portanto, a natureza da casca dos galhos não pode ser considerada um filtro limitante em relação ao tamanho corporal. No entanto, Yanoviak et al. (2012) observaram que, por sua vez, o diâmetro dos galhos funciona como um filtro que limita o tamanho corporal das formigas que ali transitam, mas apenas para formigas maiores. Essas considerações permitem inferir que diversas poneromorfas arborícolas, tais como Paraponera clavata (Fabricius, 1775) (Figura 20.3) ou N. villosa, juntamente com outras formigas não poneromorfas de grande tamanho corporal, por exemplo, Camponotus cacicus Emery, 1903 ou Cephalotes atratus (Linnaeus, 1758), utilizam sistematicamente galhos e lianas acima de certo tamanho para se deslocar entre estratos do sub-bosque e do dossel para acessar os recursos.

No dossel estruturas como galhos e lianas são fatores facilitadores para o forrageio de formigas maiores, em especial as poneromorfas. Essas estruturas servem de pontes de acesso para a exploração de numerosos recursos, tais como nectários extraflorais (NEFs), "honeydew", ou mesmo alcançar presas. Esses elementos estruturais do dossel, como lianas, galhos, epífitas, frutos e mesmo as folhas, conferem uma maior complexidade ao habitat e têm efeito sobre as áreas de ocupação

FIGURA 20.2 - Visão da estrutura da serapilheira (a) formada pela deposição de folhas e gravetos sobre a superfície do solo de uma agrofloresta, e vista em perspectiva da copa de uma árvore de uma agrofloresta a partir de sua base (Fotos: Wesley DaRocha (a) e Jacques Delabie (b)).

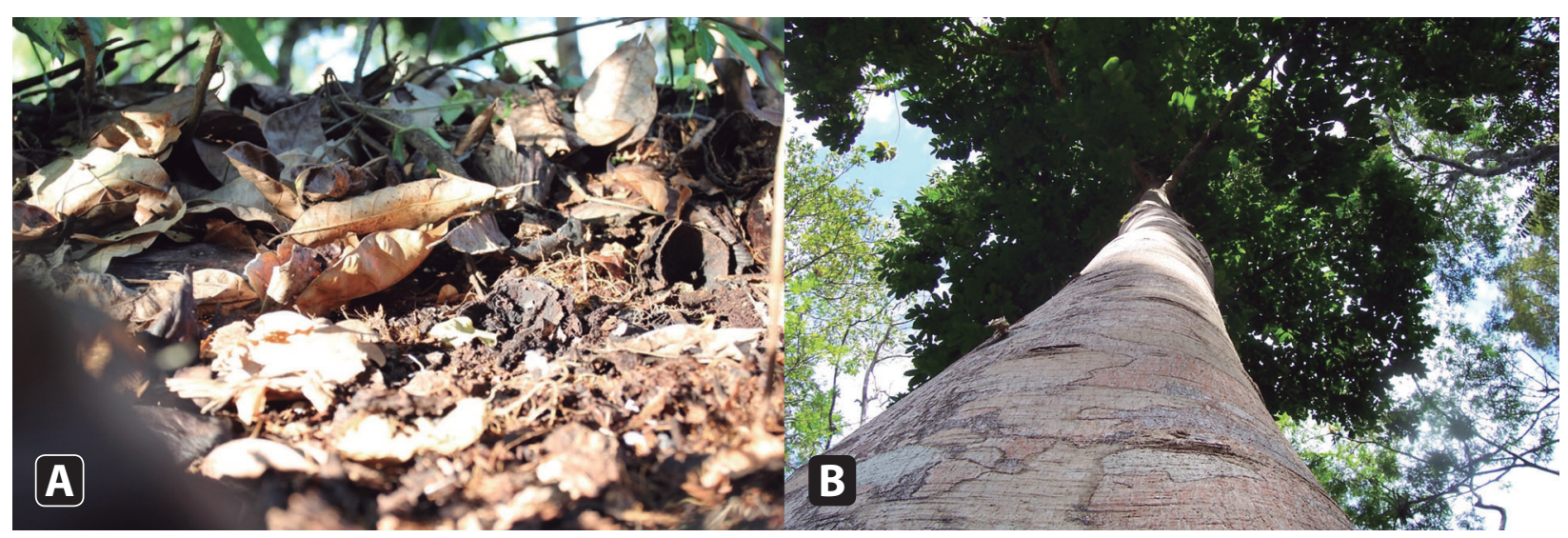


FIGURA 20.3 - Paraponera clavata (conhecida regionalmente como "tocandira", ou em inglês como "Bullet Ant") registrada sobre ramos da vegetação arbórea no bioma cerrado, Mato Grosso do Sul, transportando uma aranha (Foto: Paulo Robson de Souza).

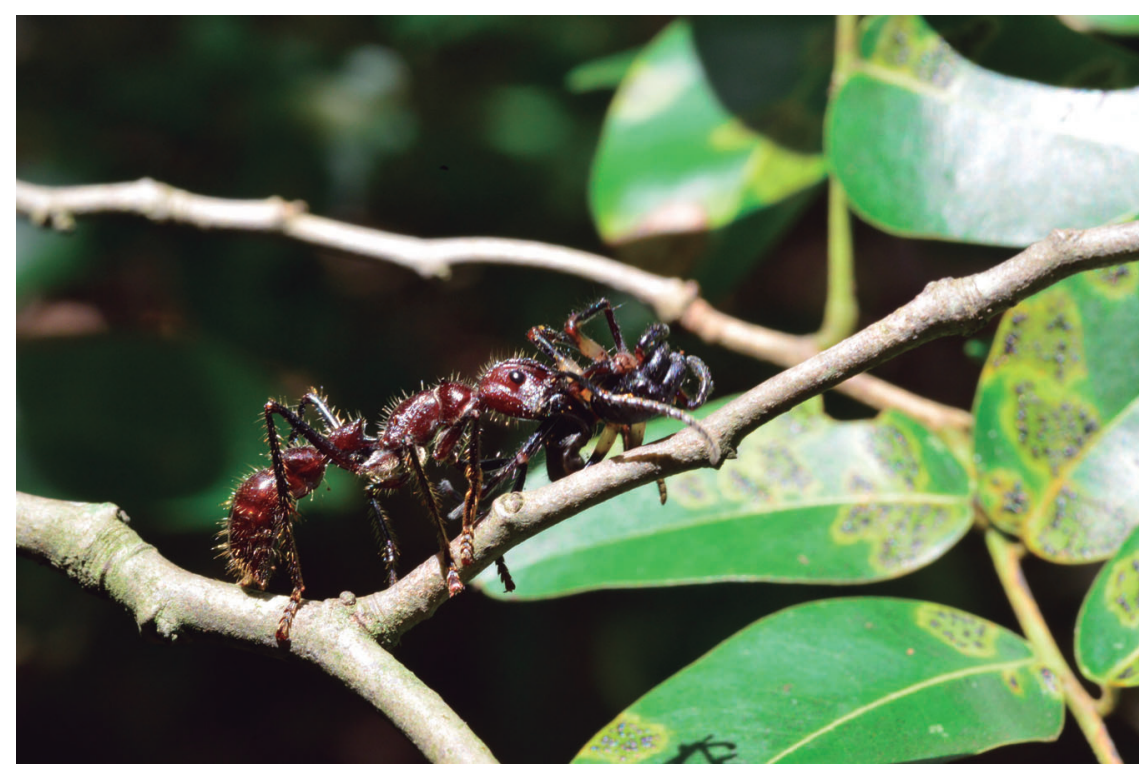

das espécies dominantes e a organização da comunidade de formigas em geral. Entretanto, como os acessos podem ser restritos como descrito acima, há poucas alternativas de uso do dossel como caminho para grandes espécies. Esta limitação resulta em uma menor disponibilidade de recursos e uma maior probabilidade de encontros entre espécies, e assim aumenta a probabilidade de ocorrer interações agonísticas no dossel onde formigas maiores seriam favorecidas diante de uma hipotética competição (YANOVIAK; KASPARI, 2000; ESPÍRITO-SANTO et al., 2012).

No dossel, as lianas têm uma importância especial como elementos que aumentam a heterogeneidade e diversidade e atuam como estruturas físicas que favorecem o acesso aos recursos para diversos animais, através do estabelecimento de conexões entre copas de árvores (DIAL et al., 2006; YANOVIAK; SCHNITZER, 2013; YANOVIAK, 2015), possibilitando o acesso aos nectários, presas e locais de nidificação (YANOVIAK; SCHNITZER, 2013). As conexões físicas entre copas de árvores vizinhas, proporcionadas por lianas, permitem a transposição dos espaços, às vezes estreitos, que existem entre as copas das árvores e que são conhecidos como "timidez de dossel" (ver NG, 1977; PUTZ et al., 1984). Além disso, lianas facilitam a conexão do dossel com os estratos inferiores como o sub-bosque e até mesmo a serapilheira. Estudos experimentais de remoção das lianas (YANOVIAK, 2015) demonstram que a ausência destas tem efeito negativo sobre a riqueza de espécies (com uma redução de $22 \%$ da riqueza média de formigas), comparativamente às áreas experimentais controle, sem remoção das lianas. Foi atribuído às Ponerinae que ocupam as árvores o papel de reguladoras da mudança na estrutura da assembleia após a remoção das lianas, principalmente através da redução da conexão física entre árvores. Esses predadores mais ou menos especializados utilizam essas conexões entre as copas, e suas distribuições locais e áreas de forrageio passam a ser dependentes dos acessos a áreas mais amplas que esses corredores entre árvores permitem alcançar.

Além das lianas, epífitas são particularmente frequentes no dossel das florestas tropicais (KITCHING et al., 1997; STUNTZ et al., 2002; YANOVIAK et al., 2004) e inúmeros estudos destacam a importância dessas plantas para a estruturação das comunidades de formigas arbóreas (DEJEAN; OLMSTEAD; SNELLING, 1995; YANOVIAK et al., 2003; DAVIDSON et al., 2003; YANOVIAK et al., 2006; YANOVIAK et al., 2011; DaROCHA et al., 2015), assim como para a formação de um dos principais microhabitats dos dosséis florestais tropicais. As epífitas apresentam-se como recursos oferecidos para nidificação de formigas arborícolas dependentes dos abrigos e dos recursos nutricionais que podem fornecer e, com 
isso, contribuem para a manutenção de uma riqueza elevada de formigas nas florestas neotropicais (YANOVIAK et al., 2011; DaROCHA et al., 2015). Segundo DaROCHA (2010) e DaROCHA et al. (2015), estudos realizados no dossel da floresta tropical úmida apontam que a assembleia de formigas poneromorfas corresponde a uma parcela importante da comunidade de formigas arbóreas, representando de $20 \%$ a $30 \%$ de todas as formigas presentes nas bromeliáceas epífitas. Em DaROCHA et al. (2015), as poneromorfas amostradas em uma única copa de árvore representam 21,3\% das espécies coletadas e 33\% dos gêneros (Tabela 20.II). Em um estudo comparativo realizado em sistemas agroflorestais cacaueiros e Mata Atlântica (DaROCHA, 2010) foi observada a seguinte representatividade para as espécies e gêneros de poneromorfas: 40\%-34,8\% (Agrofloresta com dossel entre 20 e $25 \mathrm{~m}$ de altura), 21\%-28\% (Agrofloresta com dossel superior a 25m), 14,3\%-21,7\% (Agrofloresta de sombreamento monoespecífica com cerca 20 e $25 \mathrm{~m}$ de altura) e 31\%-35,3\% (Floresta secundária madura, dossel com cerca $25-30 \mathrm{~m}$ de altura) (Tabela 20.II). Com exceção de duas espécies de Prionopelta, as demais espécies são todas de grande tamanho corporal. Ressalta-se a importância das bromélias entre as epífitas devido ao seu tamanho que facilita a nidificação de grandes espécies de poneromorfas (por exemplo, N. villosa e A. hohenbergiae). Essas espécies possuem colônias com relativamente poucos indivíduos (entre 50500 operárias), porém de grande tamanho corporal quando comparadas às grandes colônias de formigas não poneromorfas com pequenos e numerosos indivíduos dos gêneros Azteca (subfamília Dolichoderinae) ou Crematogaster (Myrmicinae), às vezes com mais de um milhão de operárias.

Essas relações entre plantas e formigas são diversas, influenciando umas às outras direta ou indiretamente. Algumas plantas podem apresentar estruturas capazes de abrigar colônias de formigas. Estruturas importantes disponíveis para formigas nidificarem, tais como as domácias que existem em plantas de diversas famílias, promovem as interações mutualísticas ou mesmo neutras entre formigas e plantas (ROSUMEK et al., 2009). Um caso clássico de domácia primária na Região Neotropical (BENSON, 1984) é o das umbaúbas (ou embaúbas), árvores do gênero Cecropia spp., e de sua associação mutualística com formigas Dolichoderinae do gênero Azteca (JOLIVET, 1986; HARADA; BENSON, 1988; DAVIDSON; FISHER, 1991). As formigas ocupam cavidades naturais do tronco das umbaúbas após ter perfurado orifícios em determinadas áreas mais finas das paredes internas e do tronco da planta. Neoponera insignis (MACKAY; MACKAY, 2010), na Costa Rica, e Neoponera luteola (Roger, 1861), no Peru, são exemplos de Ponerinae especializadas em nidificar em troncos de Cecropia (DAVIDSON; FISHER, 1991; MACKAY; MACKAY, 2010). Na América Central, diversas espécies de Pseudomyrmex constroem seus ninhos em cavidades pré-existentes nos espinhos de várias espécies de leguminosas do gênero Acacia (WARD, 1991, 1993). Diferentes tipos de domácias primárias podem existir em raízes, bulbos, tubérculos, como nas pteridófitas do gênero Solenopteris, ou espinhos, como nas Acacia da América Central. Em muitos casos, as domácias são cobertas por numerosos pêlos compridos, chamados tricômios, destinados, segundo certas interpretações, a limitar o acesso à domácia para formigas acima de certo padrão de tamanho (DAVIDSON; MCKEY, 1993). A coevolução com as plantas, traduzida pela formação de domácias por estas, parece ter ocorrido no gênero Pseudomyrmex pelo menos 12 vezes de forma independente (plantas dos gêneros Acacia, Tachigali e Triplaris), enquanto ocorreu pelo menos duas vezes no gênero Azteca (LONGINO, 1991; WARD, 1991; DAVIDSON; MCKEY, 1993a; THOMPSON, 1994). Esse fenômeno é conhecido como coevolução difusa (WARD, 1991), gerando mudanças evolutivas recíprocas entre os grupos de espécies. Portanto, as plantas implicadas exercitam pressão seletiva sobre as formigas que as habitam e vice-versa. Como exemplo de domácias secundárias (BENSON, 1984), pode ser citada a formação de uma bolsa, ou dobra laminar, na base das folhas ou nas suas laterais, como em algumas Melastomotaceae (SOLANO et al., 2003). Outras estruturas particulares da planta podem, secundariamente, oferecer abrigos para populações de formigas, como as vagens de algumas leguminosas ou formas de galhas, mas não podem ser consideradas como verdadeiras domácias. Em geral, as poneromorfas encontradas em domácias de plantas da Região Neotropical podem ser consideradas como ocupantes secundárias.

\section{Recursos alimentares determinando as comunidades de formigas no dossel}

Os recursos alimentares são vistos como importantes mecanismos reguladores da diversidade 
Tabela 20.II - Formicidae coletados em Bromeliaceae ocorrendo em uma única copa de árvore (Erythrina fusca) e em árvores de quatro sítios de coleta, RBU - Reserva Biológica de Una; CM - Fazenda Ararauna, Una; Fazenda Bonfim, Ilhéus; CABRUCA Fazenda Vera Cruz, Una; Fazenda Santa Rita, Ilhéus; DT - Derruba total, Centro de Pesquisa do Cacau, Ilhéus, Bahia.

\begin{tabular}{|c|c|c|c|c|c|}
\hline \multirow{2}{*}{$\begin{array}{l}\text { SUBFAMÍLIA } \\
\text { Espécies }\end{array}$} & \multicolumn{5}{|c|}{ Sítios de coleta } \\
\hline & Erythrina & RBU & $\mathrm{CM}$ & CABRUCA & DT \\
\hline \multicolumn{6}{|l|}{ DOLICHODERINAE } \\
\hline Azteca chartifex (Emery 1896) & & & $x$ & $x$ & \\
\hline Azteca instabilis (F. Smith, 1862) & $\mathrm{x}$ & & & & \\
\hline Azteca sp.2 & & & $\mathrm{x}$ & $\mathrm{X}$ & $\mathrm{X}$ \\
\hline Dolichoderus attelaboides (Fabricius, 1775) & & & & & $\mathrm{X}$ \\
\hline Dolichoderus bidens (Linnaeus, 1758) & & & & & $\mathrm{X}$ \\
\hline Dolichoderus lutosus (Smith F., 1858) & & & $\mathrm{x}$ & $\mathrm{x}$ & \\
\hline Dolichoderus voraginosus (MacKay, 1993) & & & $x$ & & \\
\hline Linepithema anathema Wild, 2007 & $\mathrm{X}$ & $x$ & $x$ & $\mathrm{X}$ & \\
\hline Linepithema humile (Mayr, 1868) & $\mathrm{x}$ & & & & \\
\hline Tapinoma sp.1 & & & $\mathrm{X}$ & $\mathrm{X}$ & \\
\hline \multicolumn{6}{|l|}{ FORMICINAE } \\
\hline Camponotus (Myrmobrachys) sp.12 & & & $x$ & $x$ & $\mathrm{X}$ \\
\hline Camponotus (Myrmobrachys) sp.7 & & & & $x$ & $\mathrm{X}$ \\
\hline Camponotus (Pseudocolobopsis) sp.1 & & & & & $\mathrm{X}$ \\
\hline Camponotus (Tanaemyrmex) sp. 1 & & & & $x$ & \\
\hline Camponotus atriceps (Smith F., 1858) & & & $x$ & $\mathrm{x}$ & \\
\hline Camponotus balzani Emery, 1894 & $\mathrm{X}$ & & $x$ & $\mathrm{X}$ & $\mathrm{X}$ \\
\hline Camponotus cingulatus (Mayr, 1862) & & $\mathrm{X}$ & & $\mathrm{X}$ & $\mathrm{X}$ \\
\hline Camponotus claviscapus Forel, 1899 & $x$ & & & & \\
\hline Camponotus crassus Mayr, 1862 & $x$ & & $x$ & $\mathrm{X}$ & $\mathrm{X}$ \\
\hline Camponotus egregius (Smith F., 1858) & & $\mathrm{x}$ & & & \\
\hline Camponotus fastigatus (Roger, 1863) & & $\mathrm{x}$ & $x$ & $\mathrm{X}$ & $x$ \\
\hline Camponotus rectangularis (Emery, 1890) & & $\mathrm{X}$ & & & \\
\hline Camponotus sexguttatus (Fabricius, 1793) & $x$ & & & $\mathrm{X}$ & $\mathrm{X}$ \\
\hline Camponotus sp.11 & & & $x$ & & \\
\hline Camponotus trapezoideus (Mayr, 1870) & & & $x$ & $\mathrm{X}$ & $\mathrm{X}$ \\
\hline Nylanderia fulva (Mayr, 1862) & $x$ & $\mathrm{X}$ & $x$ & $\mathrm{X}$ & $x$ \\
\hline Nylanderia sp.1 & $\mathrm{x}$ & & $x$ & $x$ & $x$ \\
\hline Nylanderia sp.3 & & $\mathrm{x}$ & $x$ & $x$ & $\mathrm{x}$ \\
\hline \multicolumn{6}{|l|}{ MYRMICINAE } \\
\hline Apterostigma sp.1 & & $\mathrm{X}$ & & $\mathrm{X}$ & $\mathrm{X}$ \\
\hline Apterostigma sp. 2 & & & & $x$ & \\
\hline Cephalotes atratus (Linnaeus, 1758) & $x$ & $\mathrm{X}$ & $x$ & $\mathrm{X}$ & $\mathrm{X}$ \\
\hline Cephalotes goeldii (Forel, 1912) & $\mathrm{X}$ & & & & \\
\hline Cephalotes minutus (Fabricius, 1804) & & & $x$ & & $\mathrm{X}$ \\
\hline Cephalotes pavonii (Latreille, 1809) & & & & $\mathrm{x}$ & \\
\hline Cephalotes pusillus (Klug, 1824) & $x$ & & & & \\
\hline Cephalotes sp.2 & & & & $\mathrm{X}$ & $x$ \\
\hline Cephalotes umbraculatus (Fabricius, 1804) & $x$ & & $x$ & & $\mathrm{X}$ \\
\hline Crematogaster acuta (Fabricius, 1804) & $\mathrm{X}$ & & $x$ & $\mathrm{x}$ & $x$ \\
\hline Crematogaster curvispinosa (Mayr, 1862) & & & $x$ & & \\
\hline Crematogaster erecta Mayr, 1866 & $\mathrm{X}$ & & $x$ & $\mathrm{X}$ & $x$ \\
\hline Crematogaster longispina (Emery, 1890) & & & $x$ & $X$ & $X$ \\
\hline
\end{tabular}




\begin{tabular}{|c|c|c|c|c|c|}
\hline \multirow{2}{*}{$\begin{array}{l}\text { SUBFAMÍLIA } \\
\text { Espécies }\end{array}$} & \multicolumn{5}{|c|}{ Sítios de coleta } \\
\hline & Erythrina & RBU & $\mathrm{CM}$ & CABRUCA & DT \\
\hline Crematogaster nigropilosa Mayr, 1870 & $x$ & $\mathrm{X}$ & $x$ & $x$ & \\
\hline Cyphomyrmex gp. rimosus & & $x$ & $x$ & $\mathrm{x}$ & $\mathrm{X}$ \\
\hline Cyphomyrmex gp. strigatus & & & & $\mathrm{X}$ & \\
\hline Cyphomyrmex transversus Emery, 1894 & $x$ & & & & \\
\hline Eurhopalothrix sp.1(nova) & $x$ & & & & \\
\hline Eurhopalothrix sp.2 & & $\mathrm{X}$ & $x$ & $x$ & $x$ \\
\hline Lachnomyrmex victori (Feitosa; Brandão, 2008) & & & & & $\mathrm{X}$ \\
\hline Monomorium floricula (Jerdon, 1852) & & & & $x$ & \\
\hline Nesomyrmex tristani (Emery, 1896) & $x$ & & $x$ & & $\mathrm{X}$ \\
\hline Pheidole asperithorax Emery, 1894 & $x$ & & & & \\
\hline Pheidole gp. Flavens sp.2 & $x$ & & & & \\
\hline Pheidole gp. Flavens sp.3 & $x$ & & & & \\
\hline Pheidole prox. cardinalis & $x$ & & & & \\
\hline Pheidole sp.1 & & & & $x$ & $x$ \\
\hline Pheidole sp.2 & & & $x$ & $x$ & \\
\hline Pheidole sp.3 & & & $\mathrm{X}$ & & \\
\hline Pheidole sp.4 & & & $x$ & & $x$ \\
\hline Pheidole sp.5 & & $\mathrm{X}$ & & $x$ & \\
\hline Pheidole sp.6 & & & & & $\mathrm{X}$ \\
\hline Pheidole sp.7 & & & $x$ & & \\
\hline Pheidole sp.8 & & & $x$ & & \\
\hline Pheidole sp.9 & & $\mathrm{X}$ & $x$ & & \\
\hline Pheidole sp.10 & & $\mathrm{X}$ & $x$ & $\mathrm{X}$ & $x$ \\
\hline Pheidole sp.11 & & $x$ & $x$ & $x$ & $\mathrm{x}$ \\
\hline Pheidole sp.12 & & & $x$ & $x$ & $x$ \\
\hline Pheidole sp.13 & & & $x$ & & \\
\hline Procryptocerus convergens (Mayr, 1887) & & & & & $\mathrm{X}$ \\
\hline Procryptocerus hylaeus (Kempf, 1951) & $x$ & & & $x$ & \\
\hline Procryptocerus spiniperdus Forel, 1899 & $x$ & & & & \\
\hline Rogeria besucheti (Kugler, 1994) & & & $x$ & $\mathrm{x}$ & $\mathrm{X}$ \\
\hline Rogeria foreli Emery, 1894 & $x$ & & & & \\
\hline Rogeria sp.2 & & & & $x$ & \\
\hline Sericomyrmex sp.1 & & & $x$ & $x$ & \\
\hline Solenopsis sp. 1 & $x$ & & $\mathrm{X}$ & $\mathrm{X}$ & $x$ \\
\hline Solenopsis sp.2 & $x$ & $x$ & $x$ & $\mathrm{X}$ & $\mathrm{X}$ \\
\hline Solenopsis sp.3 & $x$ & $\mathrm{X}$ & & $\mathrm{X}$ & $\mathrm{x}$ \\
\hline Strumigenys dolichognata (Weber, 1934) & $x$ & & & & $\mathrm{X}$ \\
\hline Strumigenys elongata (Roger, 1863) & $x$ & & & $\mathrm{x}$ & \\
\hline Strumigenys smithii Forel, 1886 & $\mathrm{X}$ & $\mathrm{X}$ & $\mathrm{X}$ & & $x$ \\
\hline Strumigenys sp. 1 & & & & & $\mathrm{x}$ \\
\hline Strumigenys sp.2 & & & & $x$ & \\
\hline Wasmannia sp.1 & & & $\mathrm{X}$ & $\mathrm{X}$ & $\mathrm{x}$ \\
\hline Xenomyrmex sp.1 & & & & & $x$ \\
\hline \multicolumn{6}{|l|}{ PSEUDOMYRMECINAE } \\
\hline Pseudomyrmex elongatus (Mayr, 1870) & & & & & $\mathrm{x}$ \\
\hline Pseudomyrmex gp. Pallidus sp.1 & $x$ & & & & \\
\hline
\end{tabular}




\begin{tabular}{|c|c|c|c|c|c|}
\hline \multirow{2}{*}{$\begin{array}{ll}\text { SUBFamílIA } & \\
& \text { Espécies } \\
\end{array}$} & \multicolumn{5}{|c|}{ Sítios de coleta } \\
\hline & Erythrina & RBU & CM & CABRUCA & DT \\
\hline Pseudomyrmex gp. Oculatus sp.1 & & & $\mathrm{x}$ & & $\mathrm{x}$ \\
\hline Pseudomyrmex gp. Sericeus & & & & & $\mathrm{x}$ \\
\hline Pseudomyrmex gracilis (Fabricius, 1804) & $x$ & & & $x$ & $x$ \\
\hline Pseudomyrmex oculatus (Smith F., 1855) & & $\mathrm{x}$ & $x$ & $x$ & \\
\hline Pseudomyrmex schuppi (Forel, 1901) & & & $x$ & & \\
\hline Pseudomyrmex tenuis (Fabricius, 1804) & $\mathrm{x}$ & & & & $\mathrm{x}$ \\
\hline Pseudomyrmex tenuissimus (Emery, 1906) & & & & $\mathrm{x}$ & \\
\hline Pseudomyrmex termitarius (F. Smith, 1855) & $x$ & & & & \\
\hline \multicolumn{6}{|l|}{ **PONEROMORFAS } \\
\hline \multicolumn{6}{|l|}{ AMBLYOPONINAE } \\
\hline Prionopelta punctulata Forel, 1909 & & & & & $\mathrm{x}$ \\
\hline Prionopelta sp.2 & & & $x$ & & \\
\hline \multicolumn{6}{|l|}{ ECTATOMMINAE } \\
\hline Ectatomma tuberculatum (Olivier 1792) & & $\mathrm{x}$ & $\mathrm{x}$ & $\mathrm{x}$ & \\
\hline Gnamptogenys haenschi (Emery, 1902) & & $\mathrm{x}$ & & & \\
\hline Gnamptogenys moelleri Forel, 1912 & $\mathrm{x}$ & & & $\mathrm{x}$ & \\
\hline \multicolumn{6}{|l|}{ PONERINAE } \\
\hline Anochetus hohenbergiae Feitosa; Delabie, 2012 & $\mathrm{x}$ & & $\mathrm{x}$ & & \\
\hline Hypoponera opacior (Forel, 1893) & $x$ & & & & \\
\hline Hypoponera sp. 1 & $x$ & $x$ & & & \\
\hline Hypoponera sp.2 & & & $x$ & $\mathrm{x}$ & \\
\hline Hypoponera sp.3 & & & $x$ & & \\
\hline Hypoponera sp.4 & & $x$ & & & \\
\hline Hypoponera sp.5 & & $\mathrm{x}$ & $\mathrm{x}$ & $\mathrm{x}$ & $\mathrm{x}$ \\
\hline Hypoponera sp.6 & & & $x$ & & \\
\hline Leptogenys arcuata (Roger, 1861) & & & $x$ & & \\
\hline Neoponera apicalis (Latreille, 1802) & & & $x$ & & $x$ \\
\hline Neoponera gp. Foetida sp. 8 & & & $x$ & $\mathrm{x}$ & \\
\hline Neoponera inversa (Smith F., 1858) & $x$ & $x$ & $x$ & $x$ & \\
\hline Neoponera moesta (Mayr, 1870) & $\mathrm{x}$ & & & & \\
\hline Neoponera unidentata (Mayr, 1862) & & & & & $x$ \\
\hline Neoponera venusta (Forel, 1912) & & $\mathrm{x}$ & & & $\mathrm{x}$ \\
\hline Neoponera villosa (Fabricius, 1804) & $x$ & & $x$ & $x$ & $x$ \\
\hline Odontomachus haematodus (Linnaeus, 1758) & $x$ & $x$ & $x$ & $x$ & $x$ \\
\hline Pachycondyla gp. Harpax sp.1 & & $\mathrm{x}$ & & $\mathrm{x}$ & \\
\hline Pachycondyla gp. Harpax sp.2 & & & $x$ & $x$ & $x$ \\
\hline Pachycondyla harpax (Fabricius, 1804) & $x$ & & & & \\
\hline Platythyrea pilosula (Smith F., 1858) & & & & $x$ & \\
\hline Platythyrea sinuata (Roger, 1860) & $x$ & & & & \\
\hline Pseudoponera gilberti (Kempf, 1960) & & & $\mathrm{x}$ & & \\
\hline Total de espécies poneromorfas & $\begin{array}{c}10 \\
(21,3 \%)\end{array}$ & $\begin{array}{c}9 \\
(31 \%)\end{array}$ & \begin{tabular}{|c|}
15 \\
$(39,5 \%)$
\end{tabular} & $\begin{array}{c}11 \\
(21 \%)\end{array}$ & $\begin{array}{c}8 \\
(14,3 \%)\end{array}$ \\
\hline Total gêneros poneromorfas & $\begin{array}{c}7 \\
(33 \%)\end{array}$ & $\begin{array}{c}6 \\
(35,3 \%)\end{array}$ & \begin{tabular}{|c|}
9 \\
$(37,5 \%)$ \\
\end{tabular} & $\begin{array}{c}7 \\
(28 \%) \\
\end{array}$ & $\begin{array}{c}5 \\
(21,7 \%) \\
\end{array}$ \\
\hline Total de espécies & 47 & 29 & 38 & 52 & 56 \\
\hline Total de gêneros & 21 & 17 & 24 & 25 & 23 \\
\hline
\end{tabular}


presente nos gradientes de dossel a serapilheira (OKSANEN et al., 1981; KASPARI, YANOVIAK, 2001). Segundo Kaspari e Yanoviak (2001), as comunidades de formigas arbóreas apresentam uma maior carência de recursos ricos em proteínas, por isso apresentam preferência por iscas proteicas se forem ofertadas simultaneamente com carboidratos. Já as formigas de serapilheira utilizam igualmente as fontes de recursos ricos em proteínas e açúcares. Essa preferência se deve ao tipo de recurso encontrado em cada habitat. No dossel existe uma disponibilidade de recursos ricos em carboidratos, como nectários extra-florais e insetos trofobiontes, e recursos nitrogenados são ativamente procurados tais como fezes de aves, porque são ricos em ureia. Já na serapilheira, as formigas encontram em seu habitat tanto recursos ricos em proteínas, tais como carcaças de animais, quanto recursos ricos em carboidratos, tais como frutos caídos no solo.

Assim, a produtividade primária elevada encontrada no dossel por ser uma região de intenso crescimento da vegetação resulta em elementos altamente favoráveis para a sobrevivência e estabelecimento de formigas. Seja por espécies de grande tamanho corporal ou espécies pequenas com enormes colônias (como as Azteca da subfamília Dolichoderinae), o dossel oferece condições térmicas, recursos alimentares ricos em carboidratos e presas em abundância para sustentar espécies dominantes e manipuladoras de territórios amplos (BLÜTHGEN; STORK, 2007; RIBEIRO et al., 2013).

Algumas das fontes de alimentos presentes na copa das árvores são:

\section{Nectários extraflorais}

Os nectários extraflorais (NEF's) estão entre os principais recursos estruturadores da comunidade de organismos nos estudos de interação inseto-planta. O néctar é uma solução aquosa rica em mono- e dissacarídeos concentrados com funções nutritivas e é secretado por algumas plantas (HEIL, 2015); esse termo designa também as excreções açucaradas de alguns insetos (DELABIE, 2001). As fontes de carboidratos ofertadas pelas plantas com NEF's e por esses insetos são recursos alimentares importantíssimos e previsíveis para as comunidades de formigas arbóreas. Isso vale para as poneromorfas que apenas forrageiam nesse ambiente, por exemplo, a Ectatomminae Ectatomma tuberculatum (Olivier, 1792) (Figura 20.4a), bem como para as que habitam o dossel, por exemplo, N. villosa. Ectatomma tuberculatum tem seu local de nidificação no solo e a entrada para o ninho é um "túnel" construído na base do tronco de uma árvore, com cerca de $20 \mathrm{~cm}$ (Figura 20.4b). Porém, apesar de nidificar no solo abaixo da serapilheira, a formiga é encontrada forrageando na copa de árvores de diferentes alturas, seja na vegetação arbustiva ou no estrato superior de dossel onde, com frequência, fica "à espreita" de pequenos artrópodes (DELABIE, 1990; DaROCHA et al., 2015). No dossel superior tropical úmido ela pode ser encontrada forrageando em epífitas da família Bromeliaceae (DaROCHA et al., 2015).

\section{Insetos trofobiontes}

O forrageio na busca por recursos ricos em carboidratos por algumas espécies de formigas

FIGURA 20.4 - (a) - Ectatomma tuberculatum patrulhando Membracidae, ocorrendo na copa de um cacaueiro (Theobroma cacao), (b) estrutura em chaminé encostada no tronco construída por E. tuberculatum para acessar o formigueiro localizado no solo, UnaBahia, fevereiro de 2015 (Foto: Wesley DaRocha).

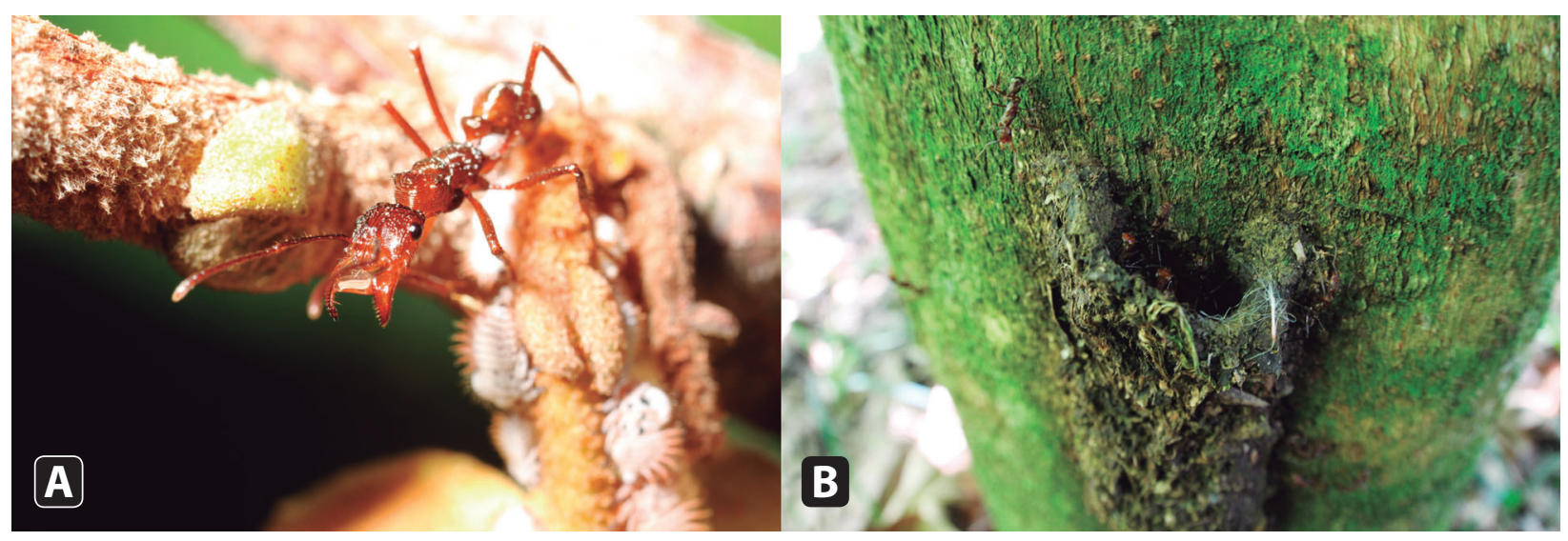


também se dá na visitação a insetos produtores de exsudatos com os quais as formigas mantêm interações mutualísticas. As mais estudadas são as relações entre Formicidae-Hemiptera, conhecidas como trofobioses (DELABIE, 2001). Os excretas dos hemípteros de numerosas famílias das subordens Sternorrhyncha e Auchenorrhyncha são conhecidos como "honeydew", e são importantes fontes de nitrogênio para as formigas (ver capítulo 24). A trofobiose ocorre em diferentes subfamílias de Formicidae, sendo de grande importância para as formigas arbóreas territorialmente-dominantes, que constituem o mosaico de formigas arbóreas (DEJEAN et al., 2007). Essas relações podem ser observadas nas subfamílias Myrmicinae, Formicinae e Dolichoderinae em que as espécies predominantemente consomem alimentos líquidos. Algumas poneromorfas também procuram esse tipo de recurso, tais como E. tuberculatum (Figura 20.4a) (DELABIE, 1990; HORA et al., 2005) e muitas Ponerinae do gênero Neoponera. Entretanto, essas poneromorfas permanecem predominantemente predadoras.

Assim como para os hemípteros mirmecófilos, esse tipo de interação também é conhecido entre Formicidae e larvas de Lepidoptera. Porém, apesar do antagonismo (ex. predação, agressão) ser grande parte da interação existente entre formigas e larvas de lepidópteros (SALAZAR; WHITMAN, 2001; ANDO; OHGUSHI, 2008), para as borboletas da Região Neotropical, a mirmecofilia é amplamente presente em apenas duas famílias (Lycaenidae e Riodinidae) (PIERCE et al., 2002; KAMINSKI et al., 2009). As secreções das larvas dessas famílias também são fontes energéticas atrativas para algumas espécies de formigas, por exemplo, a africana Oecophylla longinoda (Latreille, 1802). Isso faz com que algumas espécies de formigas levem as larvas de lepidópteros para dentro da colônia onde lhes são ofertados alimentos e proteção contra potenciais predadores e parasitas (DEJEAN; BEUGNON, 1996) em troca dessas secreções. Portanto, os NEF's e a presença destes organismos (Hemiptera e larvas de Lepidoptera) promovem a visitação das formigas sobre a vegetação. Isso, indiretamente, traz um benefício de defesa para a planta hospedeira. A manutenção dessas relações se facultativas ou obrigatórias é altamente benéfica para as larvas mirmecófilas (DeVRIES, 1991; KAMINSKI, 2008). Nestas simbioses, ambos, Hemiptera e Lepidoptera, obtêm proteção das espécies de formigas com que se relacionam contra potenciais predadores e parasitoides. Desta maneira, as interações formigas-hemípteros e formiga-lepidópteros presentes nos dosséis florestais são carreadoras do zoneamento da comunidade de formigas, isto é, mosaico.

\section{$\underline{\text { Predação }}$}

Um aspecto ainda negligenciado quanto aos recursos de dossel são as presas, normalmente herbívoros ou outros organismos visitantes foliares. Embora toda a literatura apresente a formiga como um mutualista da planta por expulsar potenciais herbívoros (ROSUMEK et al, 2009), quase nada foi feito para entender o quanto os insetos encontrados são utilizados como recurso alimentar para as formigas territoriais. Lourenço et al. (2015) demonstraram que a riqueza e abundância de herbívoros onde há espécies de formigas territoriais é menor do que onde não ocorrem essas formigas. Neste estudo, a elevada frequência de locais vazios de diversidade de insetos no dossel foi claramente associada à presença de espécies dominantes, assim como estes locais foram inversamente relacionados com a diversidade de espécies de formigas não predadoras e não territoriais (RIBEIRO et al., 2013). Segundo Gonçalves et al. (2005) e Campos et al. (2006a,b), existe um padrão inversamente proporcional de formigas e presas potencias, inclusive térmitas, no dossel de uma área de Mata Atlântica. Entretanto, pouco foi feito de fato para entender a pressão de predação exercitada pelas formigas sobre a diversidade de artrópodes de dossel, além da descrição dos padrões de distribuição.

\section{Mosaico: as comunidades de formigas arbóreas estruturam-se através das interações entre formigas territorialmente dominantes}

Numerosos estudos foram feitos sobre a estrutura das assembleias de formigas arborícolas, em particular em cacauais da África e da Região Neotropical e mais recentemente em áreas de florestas (LESTON, 1973; MAJER et al., 1994; RIBEIRO et al., 2013; CONCEIÇÃO et al., 2014) evidenciando que diversas espécies de formigas arborícolas formam o que foi batizado de mosaico de espécies dominantes. Nesses mosaicos, espécies dominantes, tais como a poneromorfa E. tuberculatum, mantêm o controle permanente sobre um amplo território (no caso, amplas extensões de plantações) através de estratégias de nidifi- 
cação, de uso de recursos e de reprodução, próprias a cada espécie que compõe o mosaico (MAJER et al., 1994; HORA et al., 2005). A possibilidade de estabelecimento de mosaicos de territórios de espécies dominantes pressupõe a ocorrência de recursos alimentares previsíveis altamente energéticos que motiva o forrageio contínuo ao longo do ano e a perenização da nidificação dessas formigas (BLÜTHGEN; STORK 2007; RIBEIRO et al., 2013). Além disso, o estabelecimento de mosaicos afeta a estrutura e riqueza da mirmecofauna encontrada na copa das árvores jovens ou velhas (MAJER et al., 1994; CAMPOS et al., 2006; CONCEIÇÃO et al., 2014).

\section{Agradecimentos}

Wesley DaRocha reconhece sua modalidade de bolsa do CNPq (processo 141751/2012-6). Jacques HC Delabie, Frederico S Neves e Sérvio P Ribeiro reconhecem suas modalidades de bolsas de pesquisa do CNPq e apoio FAPESB - FAPEMIG.

\section{Referências}

BASSET, Y.; NOVOTNY, V.; MILLER, S. E.; KITCHING, R. L. Arthropods of Tropical Forests Spatio-Temporal Dynamics and Resource use in the Canopy. Cambridge, United Kingdom: Cambridge University Press, 2003.

BASSET, Y.; ABERLENC, H. P.; DELVARE, G. Abundance and stratification of foliage arthropods in lowland rain forest of Cameroon. Ecological Entomology, v. 17, p. 310-318, 1992.

BELL, A. D.; BELL, A.; DINES, T. D. Branch construction and bud defence status at the canopy surface of a West African rainforest. Biological Journal of the Linnean Society, v. 26, p. 481-499, 1999.

BENSON, W. W. Amazon ant-plants. In: PRANCE, G. T.; LOVEJOY, T. E. (Eds.). Key Environments Amazonia. Oxford, UK: Pergamon Press, 1985. p. 239-266.

BLONDEL, J. (Ed.). Biogéographie, Approche Écologique et Évolutive. Paris: Masson, 1995.

BRANDÃO, C. R. F.; SILVA, R. R.; DELABIE, J. H. C. Neotropical ants (Hymenoptera) functional groups: nutritional and applied implications. In: PANIZZI, A. R.; PARRA, J. R. P. (Eds.). Insect Bioecology and Nutrition for Integrated Pest Management. CRC, Boca Raton, 2012. p. 213-236.
BROWN, J. H. Geographical ecology of desert rodents. In: CODY, M. L.; DIMOND, J. M. (Eds.). Ecology and Evolution of Communities. Cambridge, Massachusetts: The Belknap Press of Harvard University Press, 1975. p. 315-341.

BROWN, J. H.; LOMOLLINO, M. V. (Eds.).

Biogeography. 2. ed. Sunderland, Massachusetts: Sinauer Associates, Inc, Publishers, 1998.

CAMPOS, R. I.; VASCONCELOS, H. L.; RIBEIRO, S. P.; NEVES, F. S.; SOARES, J. P. Relationship between tree size and insect assemblages associated with Anadenanthera macrocarpa. Ecography, v. 29, n. 3, p. 442-450, 2006.

CONCEIÇÃO, E. S.; DELABIE, J. H. C.; DELLA LUCIA, T. M. C.; COSTA NETO, A. O.; MAJER, J. D. Structural changes in arboreal ant assemblages (Hymenoptera: Formicidae) in an age sequence of cocoa plantations in the south-east of Bahia, Brazil. Austral Ecology, v. 55, n. 3, 2014.

DaROCHA, W. D. As Formigas Associadas às Plantas Epífitas e sua Contribuição ao Manejo dos Cacauais do Sudeste da Bahia. [s.l.] Universidade de Santa Cruz, 2010.

DaROCHA, W. D.; RIBEIRO, S. P.; NEVES, F. S.; FERNANDES, G. W.; LEPONCE, M.; DELABIE, J. H. C. How does bromeliad distribution structure the arboreal ant assemblage (Hymenoptera: Formicidae) on a single tree in a Brazilian Atlantic forest agroecosystem? Myrmecological News, 2015.

DAVIDSON, D. W.; FISHER, B. L. Symbiosis of ants with Cecropia as a function of light regime. In: Huxley, C. R.; Cutler, D. (Eds.). Ant-Plant Interactions, Oxford Univ. Press, 1991. p. 289-309.

DAVIDSON, D. W.; COOK, S. C.; SNELLING, R. R.; CHUA, T. H. Explaining the abundance of ants in lowland tropical rainforest canopies. Science, v. 300, p. 969-072, 2003.

DAVIDSON, D. W.; MACKEY, D. The evolutionary ecology of symbiotic ant-plant relationship. Journal of Hymenoptera Research, v. 2, p. 13-83, 1993.

DEJEAN, A.; CORBARA, B.; ORIVEL, J.; LEPONCE, M. Rainforest canopy ants: the implications of territoriality and predatory behavior. Functional Ecosystems and Communities, v. 1, n. 2, p. 105-120, 2007.

DEJEAN, A.; OLMSTEAD, I.; SNELLING, R. R. TreeEpiphyte-Ant relationships in the low inundated forest of Sian Ka'an Biosphere Reserve, Quintana Roo, Mexico. Biotropica, v. 27, n. 1, p. 57-70, 1995. 
DEJEAN, A.; BEUGNON, G. Host-ant trail following by myrmecophilous larvae of Liphyrinae (Lepidoptera, Lycaenidae). Oecologia, v. 106, p. 57-62, 1996.

DELABIE, J. H. C. The ant problems of cocoa farms in Brazil. In: VANDER MEER, R. K.; JAFFÉ, K.; CEDEÑO, A. (Eds.). Applied Myrmecology : a World Perspective. Colorado, USA: Westview Press, Boulder, 1990. p. 555-569.

DELABIE, J. H. C. Comunidades de formigas das árvores nas formações florestais da América do Sul, com ênfase no sudeste da Bahia. Anais do XVI Simpósio de Mirmecologia, UFSC, Florianópolis-SC, 109-114, 2003.

DELABIE, J. H. C. et al. Communauté des fourmis des souches d'arbres morts dans trois réserves de la forêt Atlantique brésilienne (Hymenoptera, Formicidae). Ecologia Austral, v. 7, p. 95-103, 1997.

DELABIE, J. H. C. Trophobiosis between Formicidae and Hemiptera (Sternorrhyncha and Auchenorrhyncha): an overview. Neotropical Entomology, v. 30, n. 4, p. 501-516, 2001.

DELABIE, J. H. C.; BENTON, F. P.; MEDEIROS, M. A. La polydomie de Formicidae arboricoles dans les cacaoyères du Brésil: optimisation de l'occupation de l'espace ou stratégie défensive? Actes des Colloques Insectes Sociaux, v. 7, p. 173-178, 1991.

DELABIE, J. H. C.; JAHYNY, B.; NASCIMENTO, I. C.; MARIANO, C. S. F.; LACAU, S.; CAMPIOLO, S.; PHILPOTT, S. M.; LEPONCE, M. Contribution of cocoa plantations to the conservation of native ants (Insecta: Hymenoptera: Formicidae) with a special emphasis on the Atlantic forest fauna of southern Bahia, Brazil. Biodiversity and Conservation, v. 16, p. 2359-2384, 2007.

DELABIE, J. H. C.; DaROCHA, W. D.; FEITOSA, R. M.; DEVIENNE, P.; FRESNEAU, D. Gnamptogenys concinna (F. Smith, 1858): nouvelles données sur sa distribution et commentaires sur ce cas de gigantisme dans le genre Gnamptogenys (Hymenoptera, Formicidae, Ectatomminae). Bulletin de la Société Entomologique de France, v. 115, n. 3, p. 269-277, 2010.

DELABIE, J. H. C.; JAHYNY, B. A mirmecosfera animal : relações de dependência entre formigas e outros animais. O Biológico, v. 69, n. 2, p. 7-12, 2007.

DELABIE, J. H. C.; OSPINA, M. \& ZABALA, G. Relaciones entre hormigas y plantas: una introducción. Introducción a las hormigas de la región Neotropical. F. Fernández (Ed.), Instituto de Investigación de Recursos Biológicos Alexander von Humboldt, Bogotá, Colombia, 167-180, 2003.
DeVRIES, P. J. Mutualism between Thisbe irenea butterflies and ants, and the role of ant ecology in the evolution of larval-ant associations. Biological Journal of the Linnean Society, v. 43, p. 179-195, 1991.

DIAL, R. J.; ELLWOOD, M. D. F; TURNER, E. C.; FOSTER, W. A. Arthropod abundance, canopy structure, and microclimate in a Bornean lowland tropical rain forest. Biotropica, v. 38, n. 5, p. 643-652, 2006.

DJIÉTO-LORDON, C.; DEJEAN, A.; GIBERNAU, M.; HOSSAERT-MCKEY, M.; MCKEY, D. Symbiotic mutualism with a community of opportunistic ants: protection, competition, and ant occupancy of the myrmecophyte Barteria nigritana (Passifloraceae). Acta Oecologica, v. 26, p. 109-116, 2004.

DUNN, R. R.; AGOSTI; D.; ANDERSEN, A. N.; ARNAN, X.; BRUHL, C.A.; CERDÁ, X.; ELLISON, A. M.; FISHER, B. L.; FITZPATRICK, M. C.; GIBB, H; GOTELLI, N. J.; GOVE, A. D.; GUENARD, B.; JANDA, M.; KASPARI, M.; LAURENT, E. J.; LESSARD, J. P.; LONGINO, J. T.; MAJER, J. D.; MENKE, S. B.; McGLYNN, T. P.; PARR, C. L.; PHILPOTT, S. M.; PFEIFFER, M.; RETANA, J.; SUAREZ, A. V.; VASCONCELOS, H. L.; WEISER, M. D.; SANDERS, N. J. Climatic drivers of hemispheric asymmetry in global patterns of ant species richness. Ecology Letters, v. 12, n. 4, p. 324-333, 2009.

FEITOSA, R. M.; LACAU, S.; DaROCHA, W. D.; OLIVEIRA, A. R.; DELABIE, J. H. C. A giant new arboreal species of the ant genus Anochetus from Brazil (Formicidae: Ponerinae). Annales de la Société entomologique de France, v. 48, n. 3-4, p. 253-259, 2012.

FONSECA, C. R. Herbivory and the long-lived leaves of an Amazonian ant-tree. Journal of Ecology, v. 82, p. 833-842, 1994.

FONSECA, C. R.; BENSON, W.W. Ontogenetic succession in Amazonian ant trees. Oikos, v. 41, p. 2-7, 2003.

FOWLER, H. G.; DELABIE, J. H. C.; BRANDÃO, C. R. F.; FORTE, L. C.; VASCONCELOS, H. L. Ecologia nutricional de formigas. In: PANIZZI, A. R.; PARRA, J. R. P. (Eds.). Ecologia Nutricional de Insetos e Suas Implicações no Manejo de Pragas. Manole/Brasilia: CNPq, 1991.p. 131-209.

HALLÉ, F.; OLDEMAN, R. A. A.; TOMLINSON, P. B. Tropical Trees and Forests, an Architectural Analysis. Berlin: Springer-Verlag, 1978.

HEIL, M. Extrafloral nectar at the plant-insect interface: a spotlight on chemical ecology, phenotypic plasticity, and food webs. Annual Review of Entomology, v. 60, p. 213-232, 2015. 
HÖLLDOBLER, B.; WILSON, E. O. The Ants.

Massachusetts: Harvard University Press, 1990. v. N1

HOOD, W. G.; TSCHINKEL, W. R. Desiccation resistance in arboreal and terrestrial ants.

Physiological Ecology, v. 15, p. 23-35, 1990.

HORA, R. R.; VILELA, E. F.; FÉNERON, R.; PEZON, A.; FRESNEAU, D.; DELABIE, J. H. C. Facultative polygyny in Ectatomma tuberculatum (Formicidae Ectatomminae). Insectes Sociaux, v. 52, n. 2, p. 194-200, 2005.

JIMÉNEZ, E.; FERNÁNDEZ, F.; ARIAS, T. M.; LOZANO-ZAMBRANO, F. H. (Eds.). Sistemática, Biogeografía y Conservación de las Hormigas Cazadoras de Colombia. Bogotá, D. C., Colombia: Instituto de Investigación de Recursos Biológicos Alexander von Humboldt, 2008.

JOVILET, P. Ants and Plants, an Example of

Coevolution. Leiden: Backhuys Publishers, 1996.

KAMINSKI, L. A. Polyphagy and obligate myrmecophily in the butterfly Hallonympha paucipuncta (Lepidoptera: Riodinidae) in the Neotropical Cerrado Savanna. Biotropica, v. 40, n. 3, p. 390-394, 2008

KAMINSKI, L. A.; SENDOYA, S. F.; FREITAS, A. V. L.; OLIVEIRA, P. S. Ecologia comportamental na interface formiga-planta-herbívoro: interações entre formigas e lepidópteros. Oecologia Brasiliensis, v. 13, p. $27-44,2009$.

KASPARI, M. Body size and microclimate use in Neotropical granivorous ants. Oecologia, v. 96, p. 500-507, 1993.

KASPARI, M.; CLAY, N. A.; LUCAS, J.; YANOVIAK, S. P.; KAY, A. Thermal adaptation generates a diversity of thermal limits in a rainforest ant community. Global Change Biology, v. 21, p.1092-1102, 2014.

KASPARI, M.; WARD, P. S.; YUAN, M. Energy gradients and the geographic distribution of local ant diversity. Oecologia, v. 140, n. 3, p. 407-414, 2004.

KASPARI, M.; WEISER, M. D. The size-grain hypothesis and interspecific scaling in ants. Functional Ecology, v. 13, p. 530-538, 1999.

KASPARI, M.; YANOVIAK, S. P. Bait use in tropical litter and canopy ants - evidence of differences in nutrient limitation. Biotropica, v. 33, n. 1, p. 207-211, 2001.

KASPARI, M.; YANOVIAK, S. P. Biogeochemistry and the structure of tropical brown food webs. Ecology, v. 90, n. 12, p. 3342-3351, 2009.
KITCHING, R. L.; MITCHELL, H.; MORSE, G.; THEBAUD, C. Determinants of species richness in assemblages of canopy arthropods in rainforests. In: STORK, N. E.; DIDHAM, R. K.; ADIS, J. (Eds.). Canopy Arthropods. London, UK: Chapman and Hall, 1997. p. 131-150.

LATTKE, J. E. Revision of the ant genus Gnamptogenys in the New World (Hymenoptera : Formicidae). Journal of Hymenoptera Research, v. 4, p. 137-193, 1995.

LAWRENCE, R.; POTTS, B. M.; WHITHAM, T. G. Relative importance of plant ontogeny, host genetic variation, and leaf age for a common herbivore. Ecology, v. 84, p. 1171-1178, 2003.

LAWTON, J. H. Plant architecture and the diversity of phytophagous insects. Annual Review of Entomology, v. 28, p. 23-39, 1983.

LESTON, D. A Neotropical ant garden. Annals of Entomological Society of America, v. 71, p. 649-653, 1978.

LUCAS, C.; FRESNEAU, D.; KOLMER, K.; HEINZE, J.; DELABIE, J. H. C.; PHO, D. B. A mutidisciplinary approach to discrimining different taxa in the species complex Pachycondyla villosa (Formicidae). Biological Journal of the Linnean Society, v. 75, p. 249-259, 2002.

MaCKAY, W. P.; MaCKAY, E. E. The Systematics and Biology of the New World Ants of the Genus Pachycondyla (Hymenoptera: Formicidae). Edwin Mellon Press, Lewiston, 2010.

MAJER, J. D.; DELABIE, J. H. C.; SMITH, M. R. B. Arboreal ant community patterns in Brazilian cocoa farms. Biotropica, v. 26, n. 1, p. 73-83, 1994.

MARTINEZ, J. J. I. Monopolization of resources by ground-nesting ants foraging on trees in Mediterranean forests Acta Oecologica, v. 11-16, p. 6566, 2015.

NADKARNI, N. M. Good-bye, Tarzan: the science of life in the treetops gets down to business. The Sciences, v. 35 , p. $28-33,1995$.

NEVES, F. S.; QUEIROZ-DANTAS, K. S.; DaROCHA, W. D.; DELABIE, J. H. C. Ants of three adjacent habitats of a transition region between the Cerrado and Caatinga biomes: the effects of heterogeneity and variation in canopy cover. Neotropical Entomology, v. 48, p. 258-268, 2013.

NG, F. S. P. Shyness in trees. Nature Malaysiana, v. 2, n. 2, p. 35-37, 1977. 
PAOLETTI, M. G.; TAYLOR, R. A. J.; STINNER, B. R.; STINNER, D. H.; BENZING, D. H. Diversity of soil fauna in the canopy and forest floor of a Venezuelan cloud forest. Journal of Tropical Ecology, v. 7, p. 373-383, 1991.

PERFECTO, I.; RICE, R. A.; GREENBERG, R.; VAN DER VOORT, M. E. Shade coffee: a disappearing refuge for biodiversity. BioScience, v. 46, n. 8, p. 598608, 1996.

PIERCE, N. E.; BRABY, M. F.; HEATH, A.; LOHMAN, D. J.; MATHEW, J.; RAND, D. B.; TRAVASSOS, M. A. The ecology and evolution of ant association in the Lycaenidae (Lepidoptera). Annual Review of Entomology, v. 47, p. 733-771, 2002.

PUTZ, F. E.; PARKER, G. G.; ARCHIBALD, R. M. Mechanical abrasion and intercrown spacing. The American Midland Naturalist, v. 112, n. 1, p. 24-28, 1984.

RIBEIRO, S. P.; ESPÍRITO SANTO, N. B.; DELABIE, J. H. C.; MAJER, J. D. Competition, resources and the ant (Hymenoptera: Formicidae) mosaic: a comparison of upper and lower canopy. Myrmecological News, v. 18, p. 113-120, 2013.

ROBERTS, D. L.; COOPER, R. J.; PETIT, L. J. Use of premontane moist forest and shade coffee agroecosystems by army ants in western Panama. Conservation Biology, v. 14, p. 192-199, 2000.

SOLANO, P. J.; DUROU, S.; CORBARA, B.; QUILICHINI, A.; CERDAN, P.; BELIN-DEPOUX, M.; DELABIE, J. H. C. \& DEJEAN, A. Myrmecophytes of the understory of French Guianian rainforests: their distribution and their associated ants. Sociobiology, v. 41, n. 2, p. 605-614, 2003.

STUNTZ, S.; ZIEGLER, C.; SIMON, U.; ZOTZ, G. Diversity and structure of the arthropod fauna within three canopy epiphyte species in central Panama. Journal of Tropical Ecology, v. 18, p. 161-176, 2002.

TOBIN, J. E. Ecology and diversity of tropical forest canopy ants. In: LOWMAN, M. D.; NADKARNI, N. M. (Eds.). Forest Canopies. San Diego, California: Academic Press, 1995. p. 129-147.

VASCONCELOS, H. L.; DAVIDSON, D. W. Relationship between plant size and ant associates in two Amazonian ant-plants. Biotropica, v. 32, n. 1, p. 100-111, 2000.

WAY, M. J.; KHOO, K. C. Role of ants in pestmanagement. Annual Review of Entomology, v. 37, p. 479-503, 1992.
WEISER, M. D.; SANDERS, N. J.; AGOSTI, D.; ANDERSEN, A. N.; ELLISON, A. M.; FISCHER, B. L.; GIBB, H.; GOTELLI, N. J.; GOVE, A. D.; GROSS, K.; GUÉNARD, B.; JANDA, M.; KASPARI, M.; LESSARD, J. P.; LONGINO, J. T.; MAJER, J. D.; MENKE, S. B.; McGLYNN, T. P.; PARR, C. L.; PHILPOTT, S. M.; RETANA, J.; SUAREZ, A. V.; VASCONCELOS, H. L.; YANOVIAK, S. P. Canopy and litter ant assemblages share similar climate-species density relationships. Biology Letters, v. 6, n. 6, p. 769-772, 2010.

WILSON, E. O. The arboreal ant fauna of Peruvian Amazon forests: a first assessment. Biotropica, v. 19, n. 3, p. 245-251, 1987.

WILSON, E. O.; HÖLLDOBLER, B. The rise of the ants: a phylogenetic and ecological explanation.

Proceedings of the National Academy of Sciences, v. 102, p. 7411-7414, 2005.

YANOVIAK, S. P.; BERGHOFF, S. M.; LINSENMAIR, K. E.; ZOTZ, G. Effects of an epiphytic orchid on arboreal ant community structure in Panama. Biotropica, v. 43, n. 6, p. 731-737, 2011.

YANOVIAK, S. P.; SILVERI, C.; HAMM, C. A.; SOLIS, M. Stem characteristics and ant body size in a Costa Rican rain forest. Journal of Tropical, v. 28, p. 199-204, 2012.

YANOVIAK, S. P. Effects of lianas on canopy arthropod community structure. In: SCHNITZER, S. A.; BONGERS, F.; BURNHAM,R. J.; PUTZ, F. E. (Eds.). Ecology of lianas. UK: John Wiley \& Sons Ltda, 2015. p. 345-361.

YANOVIAK, S. P.; KASPARI, M. Community structure and the habitat templet: ants in the tropical forest canopy and litter. Oikos, v. 89, n. 2, p. 259-266, 2000.

YANOVIAK, S. P.; NADKARNI, N. M.; GERING, J. C. Arthropods in epiphytes: a diversity component that is not effectively sampled by canopy fogging. Biodiversity and Conservation, v. 12, p. 731-741, 2003.

YANOVIAK, S. P.; DUDLEY, R.; KASPARI, M.

Directed aerial descent in canopy ants. Nature, v. 433, 624-626, 2005.

YANOVIAK, S. P.; NADKARNI, N. M.; SOLANO, J. R. Arthropod assemblages in epiphyte mats of Costa Rican cloud forests. Biotropica, v. 36, n. 2, p. 202-210, 2006.

YANOVIAK, S. P.; FISHER, B. L.; ALONSO, A. directed aerial descent behavior in African canopy ants (Hymenoptera: Formicidae). Journal of Insect Behavior, v. 21, 164-171, 2008. 
YANOVIAK, S. P.; SCHNITZER, S. A. Functional roles of lianas for forest canopy animals. In: LOWMAN, M. D.; DEVY, S.; GANESH, T. (Eds.). Treetops at risk: challenges of global canopy ecology and conservation. New York: Springer Science+Business Media, 2013. p. 209-214.
YANOVIAK, S. P.; WALKER, H.; NADKARNI, N.

M. Arthropod assemblages in vegetative vs. humic portions of epiphyte mats in a Neotropical cloud forest. Pedobiologia, v. 48, n. 1, p. 51-58, jan. 2004.

YU, D. The structural role of epiphytes in ant gardens. Biotropica, v. 26, p. 222-226, 1994. 